\title{
Results from the OPERA experiment at the CNGS beam
}

\author{
N. Mauri ${ }^{1, a}$ on behalf of the OPERA Collaboration \\ ${ }^{1}$ INFN - Sezione di Bologna, Viale Berti Pichat 6/2, I-40127 Bologna, Italy
}

\begin{abstract}
The OPERA experiment at the Gran Sasso underground laboratory was designed to study $v_{\mu} \rightarrow v_{\tau}$ oscillations in appearance mode in the CNGS neutrino beam. Five $v_{\tau}$ candidate events have been observed, allowing to assess the discovery of $v_{\mu} \rightarrow v_{\tau}$ transitions in the atmospheric sector with a significance of 5.1 $\sigma$. In this paper the $v_{\tau}$ data analysis will be discussed, with emphasis on the background constraints obtained using dedicated data-driven control samples. Results on the search for $v_{\mu} \rightarrow v_{e}$ oscillations, on the search for sterile neutrino mixing and on the atmospheric muon charge ratio will also be presented.
\end{abstract}

\section{Introduction}

Neutrino oscillations $[1,2]$ are an experimental evidence that updated the standard framework founding most particle physics theories. The results of solar, atmospheric, reactor and accelerator experiments established that flavour mixing occurs not only in the hadronic sector, but in the leptonic sector as well. This phenomenon was discovered in 1998 through the disappearance of atmospheric $v_{\mu}$. A deficit in the solar $v_{e}$ flux was already observed since 30 years at that time. The flavour disappearance is produced with an oscillatory pattern by the quantum-mechanical interference between neutrino mass eigenstates. The leptonic flavour transitions are parametrised by the PMNS unitary matrix, through which the weak interaction eigenstates are represented as linear combinations of the mass eigenstates, and by two distinct squared mass differences, $\Delta m_{s o l}^{2}$ and $\Delta m_{\text {atm }}^{2}$, determining the oscillation frequencies at the solar and at the atmospheric scale.

The observation of flavour appearance is the last step of the discovery phase of neutrino oscillations. While the solar $v_{e}$ beam cannot be exploited for direct appearance search, due to the very low energy spectrum (well below the $\mu$ mass threshold), the atmospheric and artificial $v_{\mu}$ beams have been used for this challenging purpose in the last decade. In particular, the OPERA experiment in the CNGS beam was designed to unambiguously evidence the oscillations at the atmospheric scale through the direct $v_{\tau}$ appearance.

\section{The OPERA experiment}

OPERA [3] is a long baseline neutrino experiment located in the underground Gran Sasso Laboratory (LNGS). The direct appearance search is based on the detection of $\tau$ leptons produced in Charged Current (CC) $v_{\tau}$ interactions with a signal to noise ratio of $O(10)$. The observation of the short-lived $\tau$

\footnotetext{
a e-mail: nicoletta.mauri@bo.infn.it
} 


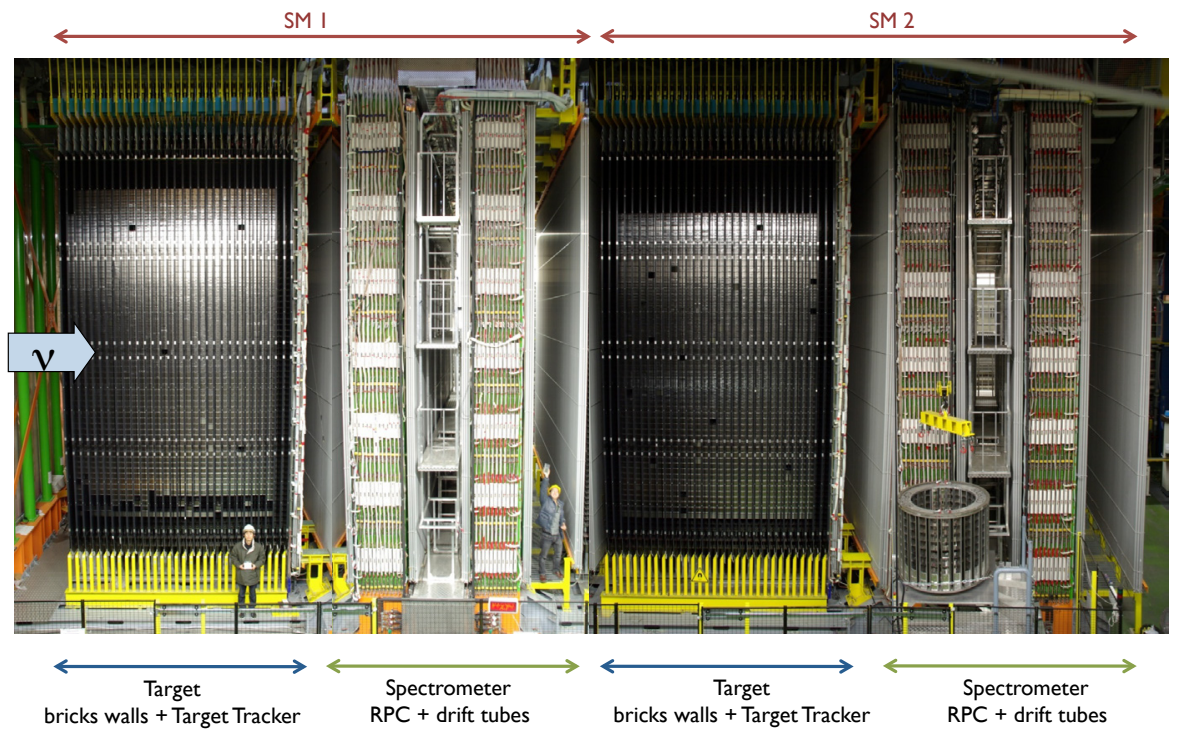

Figure 1. The OPERA detector in the Hall C at LNGS.

decay is a demanding experimental challenge, since it requires a very high tracking accuracy, $O(\mu \mathrm{m})$, integrated in a large mass to maximise the number of $v_{\tau}$ CC interactions. The OPERA experiment accomplishes these two requirements with a modular and hybrid apparatus composed by Emulsion Cloud Chambers (ECC) complemented with electronic detectors (Fig. 1). The emulsion technique allows to distinguish the three leptonic neutrino CC interations, thus OPERA has a unique capability to study $v_{e}, v_{\mu}$ and $v_{\tau}$ on event-by-event basis. In addition to its main goal, the OPERA detector at LNGS is well suited to cosmic ray physics measurements, in particular to determine the atmospheric muon charge ratio at the highest observable energies.

\subsection{The OPERA detector}

The ECC basic unit in OPERA is the "brick", a sandwich made of 56 lead plates, $1 \mathrm{~mm}$ thick, interspaced with 57 nuclear emulsion films. The submicrometre spatial resolution of the nuclear emulsion allows a precise three-dimensional reconstruction of the neutrino vertex as well as of the decay vertex associated with short-lived particles, like the $\tau$ lepton. Moreover, each brick is a compact standalone detector measuring electromagnetic showers and charged particle momentum through multiple Coulomb scattering [4]. The overall OPERA target is composed of about 150000 bricks, easily removable for the analysis, for a total mass of $1.25 \mathrm{kton}$.

In each of the two target sections, the bricks are arranged in 29 vertical "walls", transverse to the beam direction, interleaved with Target Tracker walls (TT), planes of horizontal and vertical scintillator strips. The TT trigger the read-out and allow to localise the brick containing the neutrino interaction with an accuracy of $O(\mathrm{~cm})$. Tightly packed removable doublets of emulsion films called Changeable Sheets (CS) [5] are attached downstream of each brick in a separate envelope. They serve as interfaces between the TT and the brick, in order to confirm the brick selection and to provide a 
more accurate prediction of the track position. The target section is followed by a magnetic spectrometer. A dipolar iron magnet is instrumented with RPC and drift tube detectors (Precision Trackers, PT), in order to measure the muon charge and momentum. OPERA is made of two identical Super Modules (SM) each consisting of a target section and a muon spectrometer, as shown in Fig. 1.

\subsection{The CNGS neutrino beam}

OPERA was exposed from 2008 to 2012 to the long baseline CNGS (CERN Neutrinos to Gran Sasso) $v_{\mu}$ beam, $730 \mathrm{~km}$ away from the source. The CNGS was a conventional neutrino beam produced by $400 \mathrm{GeV} / \mathrm{c}$ protons extracted from the CERN SPS and collided with a graphite target. The prompt $v_{\tau}$ contamination was negligible, $O\left(10^{-6}\right)$. The $v_{e}$ component was relatively small: in terms of CC interactions, the $v_{e}$ and $\bar{v}_{e}$ contaminations were together less than $1 \%$.

The beam was designed and optimised for $v_{\tau}$ appearance search. Differently from other neutrino beams designed to measure the disappearance signal, the mean energy of the CNGS was not tuned around the oscillation peak since at the atmospheric scale it is well below the $\tau$ production threshold (for $L=730 \mathrm{~km}$, the oscillation maximum is at $E_{v} \sim 1.5 \mathrm{GeV}$ ). The neutrino energy spectrum $\left(\left\langle E_{v_{\mu}}\right\rangle=17 \mathrm{GeV}\right)$ was set up to maximise the number of $v_{\tau} \mathrm{CC}$ interactions at LNGS, i.e. the convolution of the $\mathrm{CC}$ interaction cross section (rising with energy) and the oscillation probability (higher at low energy).

At the end of the CNGS operation, after the 2012 Run, $17.97 \times 10^{19}$ protons on target (pot) had been delivered and integrated by the OPERA detector, almost $80 \%$ of the nominal design value. The CNGS beam intensity and the total number of pot per year accumulated by OPERA are shown in Fig. 2, together with the corresponding OPERA target mass depletion.

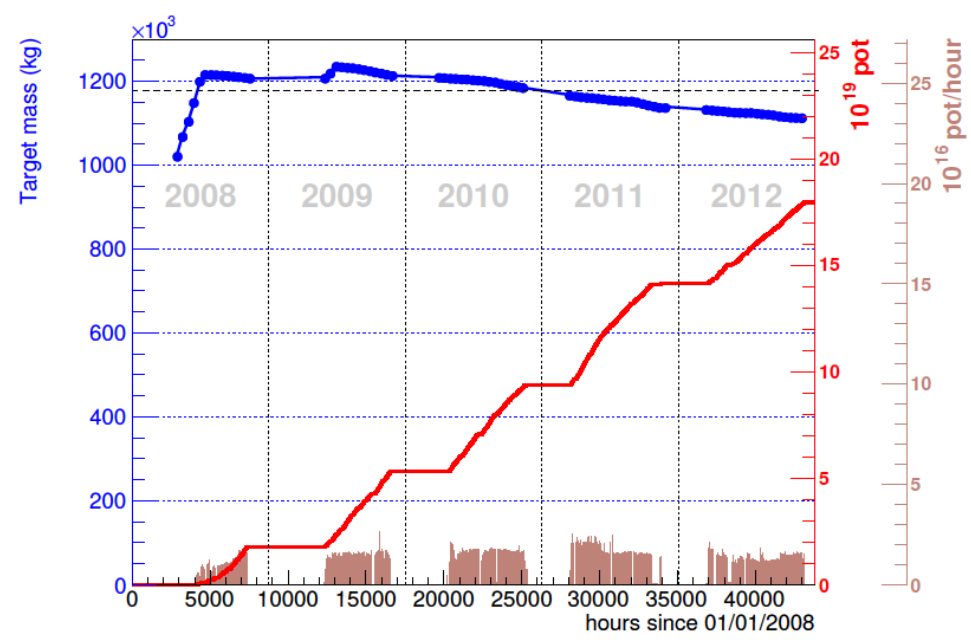

Figure 2. CNGS beam luminosity in pot and corresponding OPERA target mass evolution [12].

\subsection{Data Processing}

During the data taking from 2008 to 2012, the electronic detectors acquired both cosmic ray and CNGS beam events. The latter are selected through the timing coincidence with CERN and tagged as 
"on time" events. These are divided by an offline algorithm in "external" events, i.e. mostly muons produced by $v_{\mu}$ CC interactions in rock, and "internal" events, i.e. interactions occurring inside the OPERA target. For oscillation studies, only internal events are analysed. A total of 19505 internal interactions have been registered in 5 years of data taking. They are further classified as CC-like $(1 \mu)$ or as NC-like $(0 \mu)[6]$.

A dedicated program reconstructs the tracks in the electronic detectors and builds a 3D probability map for bricks to contain the neutrino vertex. The brick with the highest probability is extracted from the target, the CS films are detached, developed and analysed with high-speed automatic optical microscopes [7], searching for tracks compatible with the TT prediction. In case this search is unsuccessful, the brick is equipped with a new CS doublet and inserted back into the target, while the next brick in the probability map is extracted. In case of positive CS result the brick is unpacked and not replaced. The target mass evolution is shown in Fig. 2 in parallel with the CNGS integrated luminosity. The emulsion films are developed and dispatched to one of the scanning laboratories in Europe or in Japan.

The tracks found in the CS doublet are extrapolated to the most downstream film of the brick and followed upstream using predictions from the scanning in each film, until they converge to the stopping point, i.e. they are not found in three consecutive films. Vertex confirmation is obtained by scanning a large volume of $\sim 2 \mathrm{~cm}^{3}$ around the stopping point.

The final phase of the event analysis is the decay search procedure [8]. This procedure is applied to detect charged and neutral decay topologies, secondary interactions or photon conversions in the neighbourhood of the primary vertex. If a secondary vertex is found, a full kinematical analysis is performed extending the volume scanning and following the tracks also in the downstream bricks. This analysis integrates the complementary information provided by emulsions and electronic detectors, making use of the angles measured in the emulsion films, the momenta determined by multiple Coulomb scattering measured in the brick, the momenta measured by the magnetic spectrometers, and the total energy deposited in the instrumented target acting as a calorimeter [6]. The energy of photons and electrons is also estimated using calorimetric techniques [9].

\section{Neutrino oscillation results}

\section{1 $v_{\mu} \rightarrow v_{\tau}$ appearance search}

\subsubsection{Data Sets}

For the $v_{\mu} \rightarrow v_{\tau}$ appearance search the data sample consists of all the $0 \mu$ events and of the $1 \mu$ events with muon momentum below $15 \mathrm{GeV} / \mathrm{c}$. The results here reported are related to events reconstructed in the first and in the second bricks in the probability map [10]. The numbers of fully analysed events for each year of data taking are summarised in Tab. 1 .

Table 1. CNGS pot and corresponding neutrino interactions in the OPERA detector for the analysed data set.

\begin{tabular}{l|c|c|c|c|c|c}
\hline \hline & 2008 & 2009 & 2010 & 2011 & 2012 & Total \\
\hline $\operatorname{pot}\left(10^{19}\right)$ & 1.74 & 3.53 & 4.09 & 4.75 & 3.86 & 17.97 \\
$0 \mu$ & 148 & 250 & 209 & 223 & 149 & 979 \\
$1 \mu\left(p_{\mu}<15 \mathrm{GeV} / \mathrm{c}\right)$ & 534 & 1019 & 814 & 749 & 590 & 3706 \\
All events & 682 & 1269 & 1023 & 972 & 739 & 4685 \\
\hline \hline
\end{tabular}




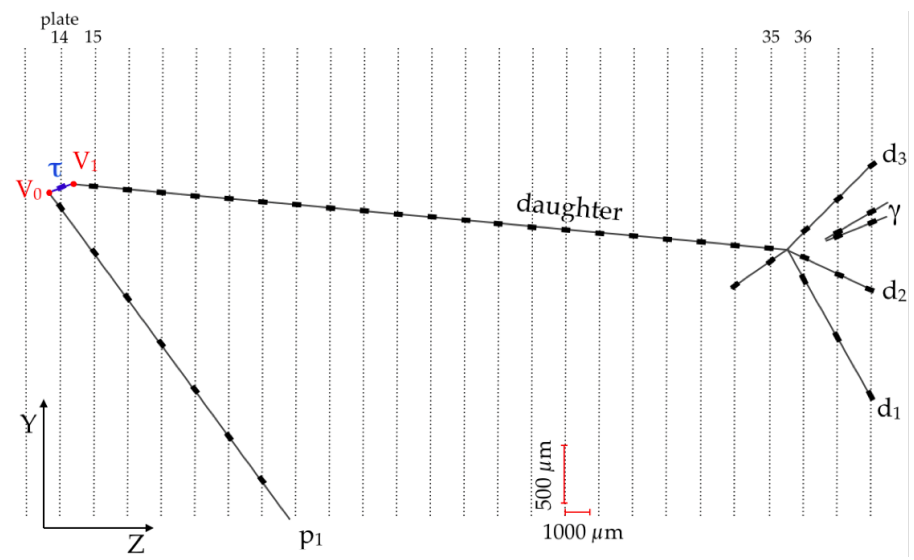

Figure 3. Display of the $5^{\text {th }} v_{\tau}$ candidate event $(\tau \rightarrow 1 h)$ in the brick (side view). The primary interaction vertex is labeled as $V_{0}$, the secondary decay vertex is labeled as $V_{1}[10]$.

After the application of the decay search procedure and the kinematical analysis, 5 events fulfill all the topological and kinematical cuts required to be $v_{\tau}$ candidates. In one of them, the $\tau$ lepton decays into a $\mu$ [11], one of them is in the $\tau \rightarrow 3 h$ decay channel [12], and three are in $\tau \rightarrow 1 h$ [13, 14]. The cuts and requirements for each decay channel are detailed in the corresponding references. Fig. 3 shows the display in the brick of the $5^{\text {th }} v_{\tau}$ candidate event, a $\tau \rightarrow 1 h$ decay.

\subsubsection{Data-driven background estimation}

The three main sources of background for the $v_{\tau}$ appearance search are charmed particle decays, hadronic interactions and large-angle muon scattering (LAS). Charmed particle decays in which the muon produced at the primary vertex in $v_{\mu} \mathrm{CC}$ interactions is not identified contribute to the background for all the $\tau$ decay channels. Hadronic re-interactions contribute only to the $\tau \rightarrow 1 h, 3 h$ decay channels, while LAS can mimic the $\tau \rightarrow \mu$ decay.

The contributions of the above mentioned processes are validated using real data samples. The observed CNGS events with charm production represent an important data set to benchmark the $\tau$ detection efficiency, due to the similar mass and decay topologies. Fig. 4 shows the distributions of the flight length of the charm candidates, the angular separation between the charmed particle and the primary muon in the beam transverse plane $(\phi)$, the impact parameters of the secondary particles with respect to the primary vertex, and the primary muon momentum for data and Monte Carlo. The expected number of charm events and the shape of the distributions are in very good agreement. The uncertainty on the charm background has been estimated to be $20 \%$ [8].

The background associated to hadronic interactions has been checked using test-beam data. $\mathrm{Nu}-$ clear fragments rates and angular distributions have been characterised up to high angle $(\tan \theta=3)$. Further constraints are given by the analysis of hadronic tracks produced in CNGS $v_{\mu}$ CC interactions. The accuracy of the expected hadronic background is at the $30 \%$ level [10]. 

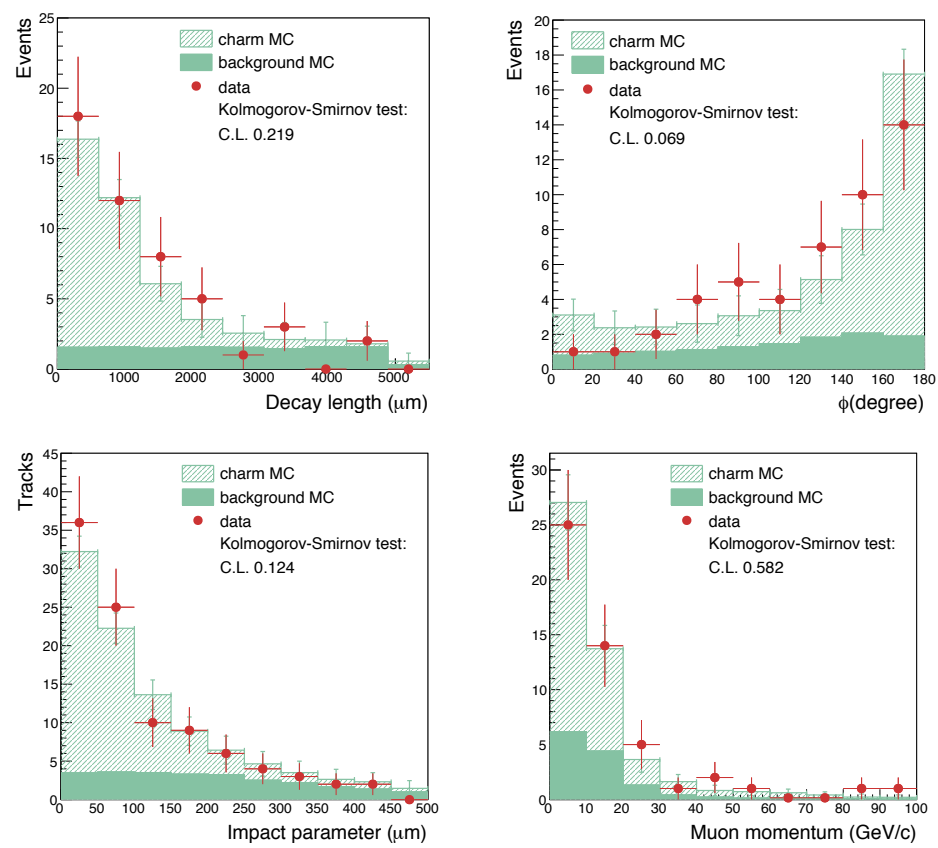

Figure 4. Shape comparison between observed $v_{\mu} \mathrm{CC}$ interactions with candidate charm decays and MC expectations. The expected background contribution is also shown (stacked histogram) [8]. Top Left: distribution of the decay length of the candidate charmed particles. Top Right: distribution of the angle between the candidate charmed particle and the primary muon in the $v$ transverse plane. Bottom Left: distribution of the impact parameters of the candidate charm daughter particles with respect to the neutrino interaction vertex. Bottom Right: distribution of muon momentum.

The LAS background contribution has been recently reevaluated and strongly reduced with respect to the value considered in the experiment proposal [15]. The LAS rate is calculated using refined Monte Carlo simulations which are benchmarked against experimental data.

The estimated background events for the analysed data set with the corresponding uncertainties are summarised in Table 2. The total expected background amounts to $0.25 \pm 0.05$ events. Details on the background expectation are given in [12].

\subsubsection{Results}

The expected numbers of $v_{\tau}$ events for each decay channel are computed assuming $\Delta m_{23}^{2}=2.44 \times$ $10^{-3} \mathrm{eV}^{2}[16]$ and maximal mixing, and are reported in Table 2 . The total expected signal amounts to $2.64 \pm 0.53$ events.

The significance of the observation of $5 v_{\tau}$ events is evaluated testing the null hypothesis, i.e. computing the probability of a background fluctuation. In this counting analysis, two test statistics are used, one based on the Fisher's method, the other one based on the profile likelihood ratio. Both methods exclude the background-only hypothesis with a significance of 5.1 standard deviations [10]. The 
Table 2. Expected signal and background events in the analysed data set [10].

\begin{tabular}{|c|c|c|c|c|c|c|}
\hline \multirow[t]{2}{*}{ Channel } & \multicolumn{4}{|c|}{ Exp. Background } & \multirow[t]{2}{*}{ Exp. Signal } & \multirow[t]{2}{*}{ Observed } \\
\hline & Charm & Hadronic re-int & LAS & Total & & \\
\hline$\tau \rightarrow 1 h$ & $0.017 \pm 0.003$ & $0.022 \pm 0.006$ & - & $0.04 \pm 0.01$ & $0.52 \pm 0.10$ & 3 \\
\hline$\tau \rightarrow 3 h$ & $0.17 \pm 0.03$ & $0.003 \pm 0.001$ & - & $0.17 \pm 0.03$ & $0.73 \pm 0.14$ & 1 \\
\hline$\tau \rightarrow \mu$ & $0.004 \pm 0.001$ & - & $0.0002 \pm 0.0001$ & $0.004 \pm 0.001$ & $0.61 \pm 0.12$ & 1 \\
\hline$\tau \rightarrow e$ & $0.03 \pm 0.01$ & - & - & $0.03 \pm 0.01$ & $0.78 \pm 0.16$ & 0 \\
\hline Total & $0.22 \pm 0.04$ & $0.02 \pm 0.01$ & $0.0002 \pm 0.0001$ & $0.25 \pm 0.05$ & $2.64 \pm 0.53$ & 5 \\
\hline
\end{tabular}

observed number of $v_{\tau}$ candidates is also compatible with the expectations from the PMNS neutrino oscillation framework.

For the CNGS baseline and energy range the rate of $v_{\tau} \mathrm{CC}$ interactions varies as $\left(\Delta m_{23}^{2}\right)^{2}$. Thus, the number of observed signal candidates is used to measure the atmospheric squared mass difference in appearance mode for the first time. Using three different approaches (profile likelihood ratio, Feldman-Cousins method, Bayesian statistics) and assuming maximal mixing, the $90 \%$ C.L. interval for $\Delta m_{23}^{2}$ is $[2.0,5.0] \times 10^{-3} \mathrm{eV}^{2}[10]$.

\subsection{Sterile neutrino mixing search}

The search for sterile neutrino mixing can be explored by OPERA in several ways. Results have been published on the search for oscillations induced by the mixing with a sterile neutrino in the $v_{\mu} \rightarrow v_{e}$ [9] and $v_{\mu} \rightarrow v_{\tau}$ [17] channels in appearance mode.

The $v_{\mu} \rightarrow v_{e}$ oscillation channel is studied thanks to the excellent capabilities of nuclear emulsions to disentangle electrons from photon conversions in identified electromagnetic showers. Here the results published in Ref. [9] are presented, which concern the analysis of 2008-2009 data, corresponding to $5.3 \times 10^{19}$ pot. The analysis of the complete data sample is ongoing and will be published in a near future.

The $v_{e}$ search is systematically applied to analysed $0 \mu$ events. A special procedure is used to select a sample of events with "shower hints", based on track multiplicity found in the CS. For the selected sample an additional scanning volume is required, from the last film of the vertex standard volume up to the most downstream film of the brick. If an electromagnetic shower is reconstructed and if the primary track initiating the shower is recognised as a single track on the vertex emulsion film, the event is classified as $v_{e}$ candidate event. Applying the above mentioned procedure, $19 v_{e}$ candidate events are observed in the 2008-2009 data set. The expected number of $v_{e}$ CC interactions due to the intrinsic beam contamination (see Sect. 2.2) is $n_{e}=19.8 \pm 2.8$ (syst.). This number is consistent with the observed $v_{e}$ candidates, showing no excess signal resulting from $v_{\mu} \rightarrow v_{e}$ oscillations. The $v_{e}$ energy spectrum is shown in Fig. 5, and compared with the expected energy spectra of the $v_{e}$ beam contamination, of the oscillated $v_{e}$ from the three-flavour model and of the background [9]. The observation is used to set limits on the oscillation parameters of the standard $3 v$ framework, with a limited sensitivity to the actual $\theta_{13}$ region [16]. An upper limit $\sin ^{2} 2 \theta_{13}<0.44$ is derived at $90 \%$ C.L.

The results are used to constrain the effective mixing parameters with a sterile neutrino. The number of $v_{e}$ candidates is compared to the expectation from an approximated two-state model parametrised in terms of two effective parameters, $\Delta m_{\text {new }}^{2}$ and $\theta_{\text {new }}$. The approximation is valid assuming $C P$ conservation, neglecting the standard oscillations, here treated as a background, and for large values of $\Delta m_{\text {new }}^{2}\left(>0.1 \mathrm{eV}^{2}\right)$. To optimise the sensitivity, an energy cut at $30 \mathrm{GeV}$ is applied. The 


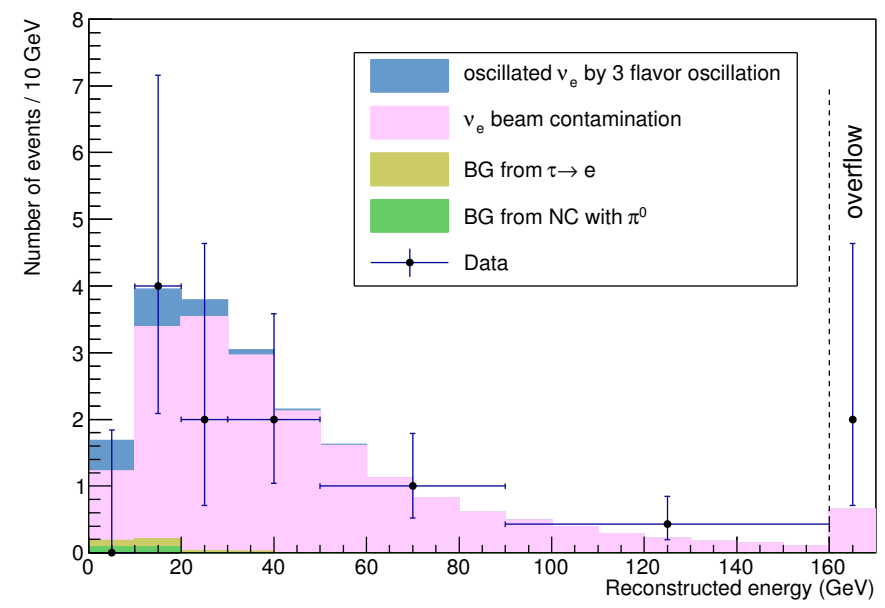

Figure 5. Energy spectrum of the observed $v_{e}$ candidate events (data points), compared with the expected energy spectra of the $v_{e}$ beam contamination, of the oscillated $v_{e}$ from the three-flavour model and of the background (histograms) [9].

number of observed events below $30 \mathrm{GeV}$ is 6 , to be compared to an expectation of $9.4 \pm 1.3$ (syst.) events. A $90 \%$ C.L. upper limit $\sin ^{2} 2 \theta_{\text {new }}<7.2 \times 10^{-3}$ is thus derived for large $\Delta m_{\text {new }}^{2}$ values. The final analysis on the complete data set will consider the so-called $3+1$ model, providing results on the 4-state mixing parameters, $\Delta m_{41}^{2}$ and $\sin ^{2} 2 \theta_{\mu e}$.

The search for sterile neutrino mixing is explored also in the $v_{\mu} \rightarrow v_{\tau}$ appearance channel. The results are presented in these Proceedings [19] and detailed in $[17,18]$. The results on $v_{\tau}$ appearance are interpreted in the context of the $3+1$ neutrino model, deriving limits on the oscillations induced by a massive sterile neutrino. Exclusion regions are obtained in the $\left(\Delta m_{41}^{2}, \sin ^{2} 2 \theta_{\mu \tau}\right)$ parameter space. The limits on $\Delta m_{41}^{2}$ are extended up to $10^{-2} \mathrm{eV}^{2}$ for relatively large mixing, $\sin ^{2} 2 \theta_{\mu \tau}>0.5$. At large values of $\Delta m_{41}^{2}\left(>1 \mathrm{eV}^{2}\right)$, marginalising over the $C P$-violating phase, values of the effective mixing parameter $\sin ^{2} 2 \theta_{\mu \tau}>0.119$ are excluded at $90 \%$ C.L.

\section{Cosmic ray physics results}

The OPERA detector located in the LNGS cavern is in a privileged location to study TeV scale cosmic rays, at an average depth of $1400 \mathrm{~m}$ of rock corresponding to $3800 \mathrm{~m}$.w.e. The minimum surface muon energy is $\sim 1 \mathrm{TeV}$ (1.4 TeV averaged over all the directions and rock depths). OPERA is the deepest experiment able to measure charge-separated atmospheric muons.

The atmospheric muon charge ratio is a highly informative observable to shed light on the primary cosmic ray composition and interactions in the very forward region. Atmospheric muons carry information on their parents, mainly pions and kaons, produced in the collisions of primaries on atmospheric nuclei.

Cosmic ray induced events were acquired by OPERA together with CNGS neutrino events and easily classified through the timing coincidence with CERN (see Sect. 2.3). In five years of data taking, more than 3 million atmospheric muon events were recorded, among which about 110000 
muon bundles, i.e. events with multiple muon tracks. Data were collected with both magnetic field polarities in order to minimise systematic errors due to misalignment.

OPERA has reported the final results on the atmospheric muon charge ratio $R_{\mu}$ using the complete statistics from 2008 up to 2012 [20]. The two data sets collected with opposite magnet polarities are combined reaching the most accurate measurement to date of $R_{\mu}$ in the $\mathrm{TeV}$ energy region. The muon charge ratio is computed separately for single muons, $R_{\mu}\left(n_{\mu}=1\right)=1.377 \pm 0.006$ (stat. $)_{-0.001}^{+0.007}($ syst. ), and for muon bundles, $R_{\mu}\left(n_{\mu}>1\right)=1.098 \pm 0.023$ (stat. $)_{-0.013}^{+0.015}$ (syst.). This is the first observation of a decrease in the charge ratio of high multiplicity events with respect to single muon events. The dilution effect in $R_{\mu}$ is expected since the multiple muon sample selects events generated by heavier primary cosmic rays and secondaries with a low value of Feynman- $x$, coming from the central region [20].

For single muons, $R_{\mu}$ is studied as a function of the vertical surface energy $E_{\mu} \cos \theta^{*}$, where $\theta^{*}$ is the zenith angle at the muon production point, fitting data to a parametrised model taking into account pions and kaons as muon parents [21]. The atmospheric muon charge ratio is measured in a wide $E_{\mu} \cos \theta^{*}$ range, from $500 \mathrm{GeV}$ up to $\sim 10 \mathrm{TeV}$, and plotted in Fig. 6 . With an average value $\left\langle E_{\mu} \cos \theta^{*}\right\rangle \simeq 2 \mathrm{TeV}$, OPERA is the magnetised experiment measuring the charge ratio at the largest vertical surface energy. The results show an increase of $R_{\mu}$ as a function of $E_{\mu} \cos \theta^{*}$ compatible with a simple parametric model where the rise is due to the increasing kaon contribution to the muon flux. The fit of OPERA and L3+C data, shown in Fig. 6, yields the fractions of charged mesons decaying into positive muons $f_{\pi^{+}}=0.5512 \pm 0.0014$ and $f_{K^{+}}=0.705 \pm 0.014$. The prompt muon component does not significantly contribute to $R_{\mu}$ up to $E_{\mu} \cos \theta^{*} \lesssim 10 \mathrm{TeV}$.

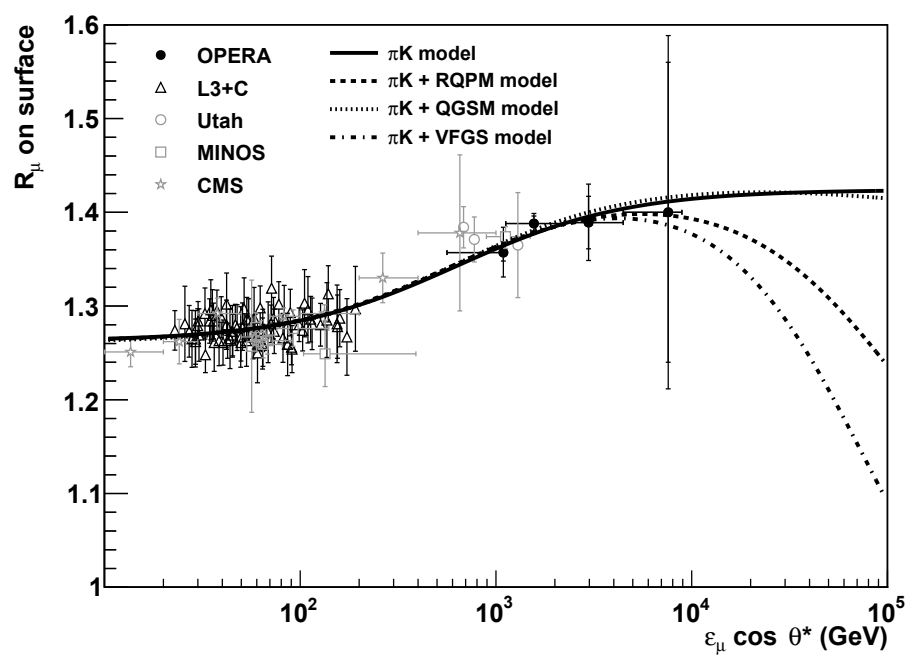

Figure 6. The atmospheric muon charge ratio measured by OPERA (black points) as a function of $E_{\mu} \cos \theta^{*}$ [20].

Taking into account the primary cosmic ray composition requires to disentangle the energy and zenith angle dependencies [21]. The fit in two dimensions $\left(E_{\mu}, \cos \theta^{*}\right)$ yields the proton excess in primary cosmic rays $\delta_{0}=0.61 \pm 0.02$ at primary energy $\left\langle E_{N}\right\rangle \approx 20 \mathrm{TeV} /$ nucleon, consistent with direct measurements of the primary composition [21], and the factor related to the associated kaon production, $Z_{p K^{+}}=0.0086 \pm 0.0004$, here determined for the first time. No similar results on the associated kaon production cross section are available to date from accelerator experiments. 


\section{Conclusions}

The OPERA experiment has observed neutrino oscillation at the atmospheric scale in appearance mode detecting $5 v_{\tau}$ candidate events. The discovery of $v_{\tau}$ appearance is assessed with a significance of $5.1 \sigma$.

The results on $v_{\mu} \rightarrow v_{\tau}$ search, compatible with the standard $3 v$ model, have been used to constrain the parameter space of oscillations induced by a massive sterile neutrino. Limits on the sterile neutrino mixing have also been derived in the $v_{\mu} \rightarrow v_{e}$ appearance channel, given the number of observed $v_{e}$ interactions.

Moreover, the OPERA detector was exploited to measure the atmospheric muon charge ratio up to the largest surface muon energy to date, $O(10) \mathrm{TeV}$. The OPERA measurement of $R_{\mu}$ in the TeV energy range determined for the first time the spectrum weighted moment $Z_{p K^{+}}$, fundamental to accurately predict the atmospheric $\bar{v} / v$ ratio.

\section{References}

[1] B. Pontecorvo, Zh. Eksp. Teor. Fiz. 33549 (1957); B. Pontecorvo, Zh. Eksp. Teor. Fiz. 34247 (1958); B. Pontecorvo, Sov. Phys. JETP 26984 (1968)

[2] Z. Maki, M. Nakagawa and S. Sakata, Prog. Theor. Phys. 28870 (1962)

[3] R. Acquafredda et al. (OPERA Collaboration), JINST 4, P04018 (2009)

[4] N. Agafonova et al. (OPERA Collaboration), New J. Phys. 14, 013026 (2012)

[5] A. Anokhina et al. (OPERA Collaboration), JINST 3 P07005 (2008)

[6] N. Agafonova et al. (OPERA Collaboration), New J. Phys. 13, 053051 (2011)

[7] N. Armenise et al., Nucl. Instrum. and Methods A 551261 (2005);

M. De Serio et al., Nucl. Instrum. and Methods A 554247 (2005);

L. Arrabito et al., Nucl. Instrum. and Methods A 568578 (2006);

C. Bozza et al., Nucl. Instrum. and Methods A 703204 (2013)

[8] N. Agafonova et al. (OPERA Collaboration), Eur. Phys. J. C 74, 2986 (2014)

[9] N. Agafonova et al. (OPERA Collaboration), JHEP 07, 004 (2013)

[10] N. Agafonova et al. (OPERA Collaboration), Phys. Rev. Lett. 115, 121802 (2015)

[11] N. Agafonova et al. (OPERA Collaboration), Phys. Rev. D 89, 051102 (2014)

[12] N. Agafonova et al. (OPERA Collaboration), JHEP 11, 036 (2013), Erratum: ibidem 04, 014 (2014).

[13] N. Agafonova et al. (OPERA Collaboration), Phys. Lett. B 691, 138 (2010)

[14] N. Agafonova et al. (OPERA Collaboration), PTEP, 101C01 (2014)

[15] A. Longhin et al., IEEE Trans. Nucl. Sc. 62, 2216 (2015)

[16] K. A. Olive et al., [Particle Data Group], Chin. Phys. C 38090001 (2014)

[17] N. Agafonova et al. (OPERA Collaboration), JHEP 06, 069 (2015)

[18] M. Tenti, proceedings of the XVII Lomonosov Conference on Elementary Particle Physics, Moscow, 2015

[19] N. Mauri, "Search for sterile neutrino mixing in the $v_{\mu} \rightarrow v_{\tau}$ appearance channel with the OPERA detector", these proceedings, ICNFP 2015

[20] N. Agafonova et al. (OPERA Collaboration), Eur. Phys. J. C 74, 2933 (2014)

[21] T. K. Gaisser, Astropart. Phys. 35, 801 (2012) 